\title{
ANÁLISE HISTOPATOLÓGICA E IMUNO-HISTOQUÍMICA (ANTíGENO NUCLEAR DE PROLIFERAÇÃO CELULAR) DE PACIENTES COM CARCINOMA CERVICAL INVASOR ANTES E APÓS RADIOTERAPIA E CIRURGIA
}

\author{
Marta Alves Rosal*, Wagner José Gonçalves, Maria Teresa de S. Alves, \\ Edmund Chada Baracat, Geraldo Rodrigues de Lima \\ Trabalho realizado no Setor de Oncocirurgia da Disciplina de Ginecologia da \\ Universidade Federal de São Paulo(EPM-UNIFESP), São Paulo-SP.
}

RESUMO - OBjETIVO. Avaliar a existência de tumor residual e o índice de proliferação celular (PCNA) em pacientes com carcinoma espinocelular do colo uterino, tratadas com a combinação de radioterapia e cirurgia.

Métodos. Avaliamos 16 pacientes com carcinoma do colo uterino atendidas no período de abril de 1986 a agosto de 1998, tratadas com irradiação pré-operatória e cirurgia. Após revisão histopatológica do material das biópsias e peças cirúrgicas, procedeu-se ao exame imuno-histoquímico para avaliar a expressão do PCNA. A imuno-expressão do PCNA foi analisada através do programa de análise gráfica por computador IMAGELAB 2.3.

Resultados. Em oito (50\%) casos detectou-se persistência de neoplasia e em outros oito (50\%) houve ausência de neoplasia residual. 0 valor médio do índice de $\mathrm{PCNA}$ anterior à radioterapia nos casos com tumor residual foi de $61,56 \%$ e, nos casos sem tumor residual, de $60 \%$. A avaliação da expressão do PCNA nas 16 biópsias prévias à radioterapia mostrou valor médio de $60 \%$. Nas peças de histerectomia com neoplasia residual a expressão do PCNA foi, em média, $74 \%$.

Conclusões. A associação de radioterapia pré-operatória e cirurgia no carcinoma do colo uterino permite adequada terapêutica em pacientes sem condições para a cirurgia radical. Outrossim, a radioterapia exclusiva apresenta percentagem relevante de insucessos detectados pela cirurgia e pela análise do PCNA.

Unitermos: Carcinoma do colo uterino. Radioterapia. Cirurgia. Tumor residual. PCNA.

\section{INTRODUÇÃO}

O câncer do colo uterino ainda permanece como importantecausademortalidade em todo o mundo. No Brasil, representa grave problema de saúde pública, assinalando-se elevada incindência em cidades como Recife, Fortaleza e São Paulo. Énecessário continuar investindo em medidas preventivas e em formas mais adequadas de tratamento!

Na atualidade, o planejamento terapêutico da neoplasia do colo uterino baseia-se na idade, nas condições clínicas da paciente, e,

*Correspondência:
Marta Alves Rosal
Rua Barão de Mogi-Guaçu, 43, Morumbi
São Paulo (SP) - CEP: 05662-050
Fone: (11) 3742-0909

principalmente, no estadiamento clínico da doença. A radioterapia é indicada para a maioria das pacientes, enquanto a cirurgia está reservada para aquelas com tumor pequeno e estádio inicial?.

A radioterapia e a cirurgia apresentam os mesmos resultados no tratamento dos casos em estádios iniciais; porém, a cirurgia seria mais apropriada para as enfermas em estádios iniciais por permitiri a eventual preservação dos ovários e ter menor interferência na vida sexual da paciente, reservando-se, para os casos avançados, a radioterapia $a^{3,45}$.

Entretanto, a combinação em várias formas da radioterapia com a cirurgia é uma prática bastante utilizada, sendo fundamental a utilização de parâmetros fidedignos na avaliação de casos assim tratados $s^{6,7}$.

Oestudo da atividade proliferativa efração de crescimento dos tumores tem indicado novas perspectivas na avaliação do diagnóstiCo, prognóstico e tratamento do câncer genital. O PCNA (antígeno nuclear de proliferação celular) já mostrou-se confiável como método de avaliação da atividade proliferativa celular em material de colo uterino ${ }^{8}$, podendo ser aplicado em tecidos fixados e incluídos em parafina, sendo identificado em células na fase de reparação do DNA e principalmente na fase $S$ do ciclo celular?

No presente estudo avaliamos a existência de tumor residual pela histopatologia; aquilatou-se, também, o índice de prolifera- 
ção celular por meio do antígeno nuclear de proliferação celular em pacientes com carcinoma espinocelular do colo uterino, tratadas com irradiação pré-operatória e cirurgia.

\section{Mítodos}

Para esse estudo, incluíram-se 16 pacientes com carcinoma espinocelular do colo uterino atendidas no período de abril de 1986 a agosto de 1998, tratadas com irradiação pré-operatória e cirurgia. Aindicação dessa modalidade terapêutica baseou-seem condições clínicas adversas das enfermas que contra-indicavam o tratamento cirúrgico radical (obesidade, cardiopatia e pneumopatia), fatores inerentes ao tumor (comprometimento parametrial importante e grande volume), além do estadiamento clínico.

Todas as informações foram obtidas pela análise retrospectiva dos prontuários das pacientes. O estadiamento clínico, baseado no sistema proposto pela Federação Internacional de Ginecologia e Obstetrícia (FIGO), teve a seguinte distribuição: 6 $(37,5 \%)$ IB, $4(25 \%)\|\mathrm{A}, 5(31,25 \%)\| \mathrm{B}$ e I (16\%) IIIB.

Atécnica e a dose da radioterapiaforam estabelecidas de acordo com as características do tumor, as condições clínicas da paciente e os dados do exame físico. Após o tratamento radioterápico, em 15 pacientes realizou-se histerectomia total abdominal e salpingooforectomia bilateral. Em duas complementou-se com colpectomia parcial. Em uma paciente, por dificuldades técnicas, fez-se apenas histerectomia subtotal.

O estudo histopatológico dos espécimes obtidos pela biópsia antecedendo a radioterapia e das peças obtidas na cirurgia foram realizados no Departamento de Patologia da UNIFESP - EPM. Todos os casos apresentavam o mesmo tipo histopatológico: carcinoma espinocelular, sendo quatro casos bem diferenciados $(\mathrm{Gl})$ e 12 casos moderadamente diferenciados (GII).
Após revisão histopatológica de todo o material proveniente das biópsias e peças cirúrgicas para confirmação diagnóstica e seleção das áreas mais representativas do tumor, procedeu-se ao exame imuno-histoquímico para avaliar a expressão do PCNA pelatécnica estrepto-avidina-biotina-peroxidase para anticorpo monoclonal anti-PCNA (clone PClO).

A técnica imuno-histoquímica foi processada no Laboratório de Imunopatologia e Técnicas Especiais do Departamento de Patologia da UNIFESP - EPM, sendo aplicada no material proveniente de todas as biópsias do colo antes de qualquer terapêutica e no material oriundo de peças cirúrgicas que apresentaram tumor residual.

A análise da imuno-expressão do PCNAfoi efetuada através do programa de análise gráfica por computador IMAGELAB 2.3. A expressão foiconsiderada positivaquando onúcleodacélula apresentava coloração castanha e negativo na sua ausência. Obteve-se o índice de células com imuno-expressão positiva pela relação entre o número de células positivas e o total de células, multiplicado por 100.

\section{Resultados}

A avaliação da peça cirúrgica pela histopatologia revelou a presença de neoplasia residual em oito casos (50\%) e ausência de neoplasia em outros oito casos (50\%).

Dos oito casos com neoplasia residual, quatro $(25 \%)$ receberam dose parcial de radioterapia (média de 4500 cCy) e quatro (25\%) receberam dose total (média de 7000 cCy). Já aqueles sem neoplasia residual, cinco $(31,25 \%)$ receberam dose total e três $(18,75 \%)$ dose parcial.

O intervalo de tempo médio entre o fim da radioterapia e a cirurgia foi de 3,5 meses, variando de I a 6 meses.

A avaliação da expressão do PCNA nas 16 biópsias prévias à radioterapia mostrou valor médio de $60 \%$. Nas peças de histe- rectomia com neoplasia residual a expressão do PCNA foi, em média, 74\%.

O valor médio do PCNA anterior à radioterapianos oito casos que apresentaram tumor na peça operatória foi de $61,56 \%$ e, nos casos sem tumor residual, foi de $60 \%$.

\section{Dıscussão}

A radioterapia e a cirurgia permanecem ainda como as melhores armas no tratamento do carcinoma do colo uterino. A indicação de uma ou de outra modalidade terapêutica exige cuidadosaavaliaçãoda paciente, considerandose sempre a idade, o estado clínico, a extensão da doença e a presença de complicações².

As pacientes envolvidas nesse estudo eram, na sua maioria, potencialmente operáveis se vistas pelo estadiamento clínico. Porém, fatores clínicos adversos ou inerentes ao tumor impediram a execução do tratamento cirúrgico radical. Assim, optouse pela radioterapia como abordagem preliminar e reavaliação subseqüente.

Em oito (50\%) casos, a obesidade foi a contra-indicação para a cirurgia radical e a radioterapianeoadjuvante permitiu uma cirurgia subsequente de menor porte. Em dois casos $(12,5 \%)$, o tumor era de grande dimensão ( $>4 \mathrm{~cm})$, necessitando inicialmente reduzir seu volume pela radioterapia para torná-los mais facilmente operáveis. Em três casos (18,75\%), além da obesidade, o estadiamento foi considerado e, em outros seis (37,55\%), face ao comprometimento parametrial, o estadiamento constituiu a indicação para o tratamento combinado.

Nos nossos resultados encontramos valores elevados de neoplasia persistente quando comparados com dados da literatu$\mathrm{ra}^{10,11,12,13}$. Dototal, oito (50\%) pacientes apresentaram neoplasia após a radioterapia. Isso pode ser explicado pela heterogeneidade do grupo e o pequeno tamanho da amostra.

Das nove pacientes que receberam dose total de radioterapia, em cinco 
ROSAL MA et AL.

$(31,25 \%)$ a peça cirúrgica era isenta de neoplasia e quatro (25\%) ainda continham tumor. Se fosse possível avaliar previamente a presença de fatores com capacidade de predizera resposta à radioterapia, poder-seia indicar mais precocemente a cirurgia.

No entanto, como se vê, três enfermas (18,75\%) não apresentavam neoplasia apenas com dose parcial de radioterapia. Sem dúvida, tratavam-se de tumores com boa radiossensibilidade. Entretanto, se estas doentes tivessem recebido dose total, certamente estaríamos aumentando a morbidade, sem qualquer benefício no tratamento.

Sabe-se que a simples presença de tumor em peça cirúrgica após a radioterapia, não significa viabilidade das células neoplásicas, pois, eventualmente, o tempo para realização da cirurgia é precoce, impedindo a completa necrose tumoral.

Entretanto, o intervalo médio entre a radioterapia e a cirurgia em nossa casuística foi de 3,5 meses. Utilizou-se o PCNA para comprovar a atividade proliferativa das células nas peças com tumor residual, bem como para avaliar o eventual efeito da radioterapia na proliferação do tumor.

A utilização do PCNA como método de avaliação de atividade nuclear é bastante freqüente, emvirtude da sua comprovada eficácia. Emalgunstrabalhos queavaliarama proliferação celular em carcinoma espinocelular do colo, o PCNA mostrou-se bom marcador $8,14,15$.

Na nossa casuística, os 16 casos expressaram o PCNA com valores que variaram de 27,91\% a 89,93\% (média de 60\%) das células, havendo concordância com dados da literatura. Após a radioterapia, nos casos em que havia neoplasia residual, o PCNA mantevese positivo (média de 74\%), ratificando a presença de atividade celular. Em quatro casos houve aumento no índice de PCNA após a irradiação e, em dois, houve diminuição.

A radiossensibilidade dos tumores é determinada, entre outros fatores, pela fração de crescimento do tumor, grau de diferenciação celular e distribuição das células nas diferentes fases do ciclo celular. Portanto, a aplicação da radioterapia sem considerar esses fatores, deverá, sim, produzir diferentes resultados ${ }^{16,17}$.

$O$ conhecimento da radiossensibilidade, ou da resposta terapêutica à radioterapia, só foi possível nesses casos com a realização da cirurgia, reavaliando o tratamento preliminare, eventualmente, encerrando a terapêutica radioterápica.

Fica claro a importância da não padronização do tratamento, baseando-se apenas em fatores como estadiamento, condições clínicas e idade, sendo necessário o conhecimento de fatores envolvidos na radiossensibilidade dos tumores.

Com uma amostra mais homogênea, a avaliação do efeito da radioterapia sobre o tumor e sua atividade proliferativa teria grande utilidade na abordagem dessas pacientes. Assim, poder-se-á em futuro próximo estabelecer critérios de seleção de pacientes que se beneficiarão das terapêuticas cirúrgica ou radioterápica ou ainda da combinação de ambas, como melhor opção de tratamento.

\section{Conclusões}

O estudo histopatológico das peças de histerectomia após o tratamento radioterápico desvelou a presença de neoplasia em 50\% dos casos, sendo $25 \%$ nas mulheres que haviam recebido dose total de radioterapia e 25\% nas que receberam dose parcial. Poder-se-ia afirmar que a simples presença de neoplasia residual nas peças não necessariamente significa tumor com capacidade biológica de progressão einvasão. Todavia, a cirurgia após a radioterapia desvelou neoplasia geralmente insuspeita na propedêutica clínica e subsidiária. Por outro lado, a subseqüente análise do PCNA comprovou a percentagem relevante de insucessos. Outrossim, a associação de radioterapia préoperátoria e cirurgia no carcinoma do colo uterino permitiua adequada terapêutica de pacientes sem condições para o tratamento cirúr- gico radical. Finalmente, nas neoplasias em estádios avançados, nos quais seriam de tratamento exclusivamente radioterápico, esta modalidade de tratamento surpreendeu os insucessos, ou seja, casos possivelmente com menor radiossensibilidade.

\section{SUMMARY}

\section{Histopathological AND IMMUNOHISTO- CHEMICAL ANALYSIS (PROLIFERATING CELL NU- CLEAR ANTIGEN) OF PATIENTS WITH INVASOR CERVICAL CARCINOMA BEFORE AND RADIOTHE- RAPY AND SURGERY}

OBIECTNE. To search for residual disease and to analyse the Proliferating Cellular Nuclear Antigen (PCNA) status, in patients with cervical squamous cell carcinoma, treated with both radiotherapy and surgery.

METHODS. Histologicalslidesfrom I 6patients with uterine cervix cancer, treated between April 1986 and August 1998, with preoperative radiotherapy and surgery, were reviewed. PCNAimunohistochemicalreactivity of these samples was evalued, using the IMAGELAB 2.3 computer image analysis system.

RESULTS. Residual carcinoma were found ineight cases (50\%) and no malignantfeatures was found in eight cases (50\%). The mean value of PCNA before radiotherapy in patients with residual cancer was $61.56 \%$ and in cases without residual cancer was $60 \%$. Its expression before radiotherapy was between $27.91 \%$ and $89.93 \%$ (60\% average), while after radiotherapy it varied between $55.80 \%$ and $86.73 \%$ (74\% average).

CONCLUSIONS. The association between preoperative radiotherapy followed by surgery is adequated to treat patients with cervicalcancer, when radicaltreatmentisnot possible. Meanwhile, exclusive radiotherapy shows a significantfailure rate, detected after surgery and PCNA analysis. [Rev Assoc Med Bras 2002; 48(I): 32-5]

KeY wORDS: Carcinoma cervical. Radiotherapy. Surgery. Residual neoplasia. PCNA. 


\section{REFERÊNCIAS}

I. Muir C, Waterhouse J, Mack T, Powell J, Whelan S. Cancer incidence in five continents. Lyon: International Agency for Research on Cancer; 1987. (IARC Scientific Publications n.88)

2. DiSaia P, Creasman W. Cáncer cervical invasor. In: Di Saia P, Creasman W, Mendizábal JA editores. Oncologia ginecológica clínica. $4^{a}$ ed. Madrid: Mosby/Doyma Libros, 1994. p.58-125.

3. Seski JC, Dioknoac N. Bladder dysfunction after radical hysterectomy. Am J Obstet Gynecol 1977; 128:6.

4. Creasman WT, Soper IT, Clarke-Pearson D. Radical hysterectomy as therapy for early carcinoma of the cervix. Am J Obstet Gynecol 1986: I55:964-9.

5. Eifel PJ, Burke TW, Delclos L, Wharton JT Oswald MJ. Early stage I adenocarcinoma of the uterine cervix; treatment results in patients with tumors $<=4 \mathrm{~cm}$. In diameter. Gynecol Oncol 1991; 41:199-205.

6. Einhorn N, Patek E, Sjoberg B. Outcome of different treatment modalities in cervix carcinoma stage IB and IIA. Cancer 1985; 55:949-55
7. Calais G, Floch OI, Chauvet B, ReynaudBougnox A, Bougnoux P. Carcinoma of the uterine cervix stage $\mathrm{IB}$ and early stage II: Prognostic value of the histological tumor regression after initial brachytherapy. Int J Radiat Oncol Biol Phys 1989; 17:1231-59.

8. Raju GC. Expression of the proliferating cell nuclear antigen in cervical neoplasia. Int J Gynecol Pathol 1994; 13:337-41.

9. Haerslev T, Jacobsen GK. Microwave processing for immunohistochemical demonstration of proliferating cell nuclear antigen (PCNA) in formalin-fixed and paraffin-embedded tissue. APMIS 1994; 102:395-400.

I 0. Parker RT, Wilbanks GD, Yowell RK, Carter FB. Radical hysterectomy and pelvic lymphadenectomy with and without preoperative radiotherapy for cervical cancer. Am J Obstet Gynecol 1967; 99:933-43.

I I. Coelho FRG. Carcinoma espinocelular do colo uterino submetido a cirurgia radical isolada ou em combinação com radioterapia. São Paulo, 1995. [Dissertação] Faculdade de Medicina da Universidade de São Paulo.

12. Rampone Jf, Klem Y, Kolstad P. Combined treatment of stage IB carcinoma of the cervix. Obstet Gynecol 1973; 41:163-7.
13. Mundt AJ, Waggoner S, Herbst A, Rotmensch J. Preoperative intracavitary brachytherapy in early-stage cervical carcinoma. Am J Clin Oncol 1999; 22:73-7

| 4. Wistuba I, Roa I, Araya IC. Proliferating cell nuclear antigen in cervical cancer and precursor lesions. Rev Med Chil 1995; I 23: | 077-83.

1 5. Kennedy AS, Raleigh JA, Perez GM, Calkins DP, Thrall DE, Novotny DB et al. Proliferation and hypoxia in human squamous cell carcinoma of the cervix: first report of combined immunohistochemical assays. Int J Radiat Oncol Biol Phys 1997; 37:897-905.

16. Yu JM, Zhang H, Wang SQ, Miao HQ, Yang $\mathrm{LH}$, Chen YT et al. DNA ploidy analysis of effectiveness of radiation therapy for cervical carcinoma. Cancer 1991; 68:76-8.

17. Tubiana M. The kinetics of tumour cell proliferation and radiotherapy. $\mathrm{Br} J$ Radiol 1971: 44:325-47

Artigo recebido: 15/12/2000 Aceito para publicação: | 9/06/200 |

\section{IMAGEM EM MEDICINA}

Participe da nova seção da Ramb. É um espaço aberto ao leitor, que poderá enviar material de interesse educativo como fotos, ilustrações e exames, acrescido de três linhas explicativas contendo ainda nome do autor e serviço onde foi realizado.

O material poderá ser enviado para a

Rua São Carlos do Pinhal, 324 - Cep 01333-903

S. Paulo - SP - E-mail: ramb@amb.org.br 